\title{
IY Ursae Majoris: Accretion disc evolution after superoutburst
}

\author{
V. Stanishev ${ }^{1, \star}$, Z. Kraicheva ${ }^{1, \star}$, H. M. J. Boffin ${ }^{2, \star}$, and V. Genkov ${ }^{1, \star}$ \\ 1 Institute of Astronomy, Bulgarian Academy of Sciences, 72 Tsarighradsko Shousse Blvd., 1784 Sofia, Bulgaria \\ 2 Royal Observatory of Belgium, Avenue Circulaire 3, 1180 Brussels, Belgium
}

Received 27 July 2000 / Accepted 15 December 2000

\begin{abstract}
CCD photometry of the newly discovered eclipsing dwarf nova IY Ursae Majoris is used to study the physical properties of the accretion disc in the late decline and quiescence stages. Eclipse mapping analysis shows that in these stages the accretion disc is cool with approximately flat radial brightness temperature distribution and $T_{\mathrm{BR}} \sim 5000-5500 \mathrm{~K}$. The hot spot is found to lie close along the stream trajectory at distance $0.36 a$ and $0.25 a$ from the disc center in February and March. The orbital hump strength decreases by $\sim 30 \%$ in a month. Taking the smallest possible size of the hot spot, we derive an upper limit for its brightness temperature, $\sim 15900 \mathrm{~K}$ and $\sim 13800 \mathrm{~K}$, respectively.
\end{abstract}

Key words. accretion, accretion disc - binaries: eclipsing - stars: individual: IY UMa - dwarf nova, cataclysmic variables

\section{Introduction}

An intensive photometric monitoring of the recently discovered dwarf nova Tmz V85 (Takamizawa 1998) was started after January 13, 2000 when Schmeer (2000) reported a new outburst. The star has been classified as a deep eclipsing SU UMa type dwarf nova by Uemura et al. (2000) and got the designation IY UMa (Samus 2000). Using numerous long photometric series covering the whole superoutburst, Patterson et al. (2000) determined the system parameters: orbital period $P_{\text {orb }}=$ 0.0739092 , masses of the two components $M_{1}=0.68 M_{\odot}$. and $M_{2}=0.10 M_{\odot}$, inclination $i=85.9^{\circ}$ and distance $D=190$ pc. During the superoutburst the star showed both "normal" and "late" superhumps with a period of $\sim 0$ d. 07583 .

Here we report our time-resolved photometric observations of IY UMa started about the end of the late superhumps stage and continued after the accretion disc shrank back in quiescence.

\section{Observations and data reduction}

The observations of IY UMa were carried out at Rozhen Observatory with the 2.0-m telescope and its attached

\footnotetext{
Send offprint requests to: Z. Kraicheva

* e-mail: vall@astro.bas.bg (VS),

e-mail: zk@astro.bas.bg (ZK),

e-mail: henri.boffin@oma.be (HMJB),

e-mail: nao@mail.orbitel.bg (VG).
}

Photometrics $1024 \times 1024$ CCD camera. The star was observed in the Johnson $V$ band for three nights, with an exposure time of $60 \mathrm{~s}$ on February 6 and March 11, 2000 and with $90 \mathrm{~s}$ on March 30, 2000. In addition, $B$ exposures were regularly taken during the nights. In each case, there was a $\sim 15 \mathrm{~s}$ dead-time. In total, seven eclipses were covered: three on February 6 and March 11 and one on March 30. After de-biasing and flat-fielding the photometry was done with the standard DAOPHOT procedures (Stetson 1987). On February 6 and March 30 the seeing was $\sim 2^{\prime \prime}$ and we performed PSF fitting photometry, which allowed to estimate the magnitudes of the faint NE companion: $V=19.5$ and $B-V=0.69$. The data on March 11 were processed using aperture photometry including this star and subtracting its flux afterwards. For all the observations the stars ID 9 with $V=17.633 \pm 0.037$ and $B-V=1.145 \pm 0.027$ and ID 10 with $V=17.844 \pm$ 0.016 and $B-V=0.620 \pm 0.136$ from the list of Henden (2000) served as a comparison star and check, respectively. The instrumental $b-v$ colors were transformed to standard ones in the usual way with a second-order extinction term $k_{b-v}^{\prime \prime}=-0.035$ included. All the $B$ frames were taken outside eclipse, therefore the $B-V$ colors are representative for these orbital phases only. The $B-V$ color was found to be constant with the orbital phase and averages $\sim 0.0$ and $\sim+0.22$ for February and March observations, respectively. These values were used to convert the instrumental $v$ band measurements to standard ones. The magnitudes were converted to fluxes using the absolute calibration of the $U B V$ system (Straižys 1977). 


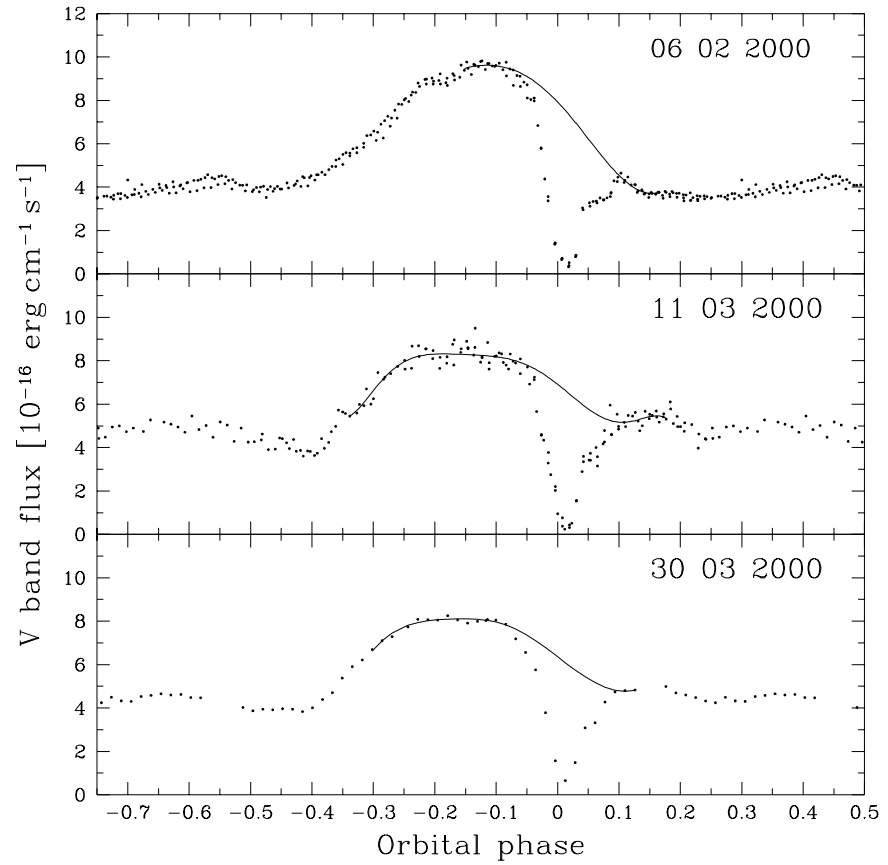

Fig. 1. Photometric light curves, folded with the orbital period $P_{\text {orb }}=0$ d 0739092 . The low order polynomial fits to the outof-eclipse data are also shown

\section{Accretion disc eclipse mapping}

In Fig. 1 the light curves are shown, folded with the ephemeris determined by Patterson et al. (2000). The analysis of these curves was performed using the system parameters determined by Patterson et al. (2000). To reconstruct the accretion disc $V$ band brightness distribution we used the eclipse mapping method (Horne 1985) and the Maximum Entropy technique as described by Skilling \& Bryan (1984) with a full azimuthal floating default image (Horne 1985). Since our data were obtained with exposure times longer than the WD ingress and egress ( $\sim 35$ s, Patterson et al. 2000), we were unable to decompose the light curves into their individual components: WD, hot spot and accretion disc. Thus, the curves had to be analysed without subtracting the WD and hot spot flux. Instead, we accounted for the variation of the hot spot flux with the orbital cycle by first fitting the out-of-eclipse data with a low-order polynomial (shown with full lines in Fig. 1), then normalizing the data to this fit and finally rescaling the data to the expected hump flux at zero phase. The curve obtained on March 30 was excluded from the analysis because of its long exposure time, hence its very poor orbital phase resolution. The final curves and the fits obtained are shown in Fig. 2 and it can be seen that the hot spot egress phase changes from $\sim 0.09$ in February to $\sim 0.075$ in March. The hot spot egress phase obtained from the curve not included in the reconstruction (taken on $30 \mathrm{March}$ ) coincides with that determined from the March 11 data. It seems also that in February the white dwarf and hot spot are eclipsed simultaneously or at least the hot spot is eclipsed very close after the white dwarf, while in March the ingress of the two
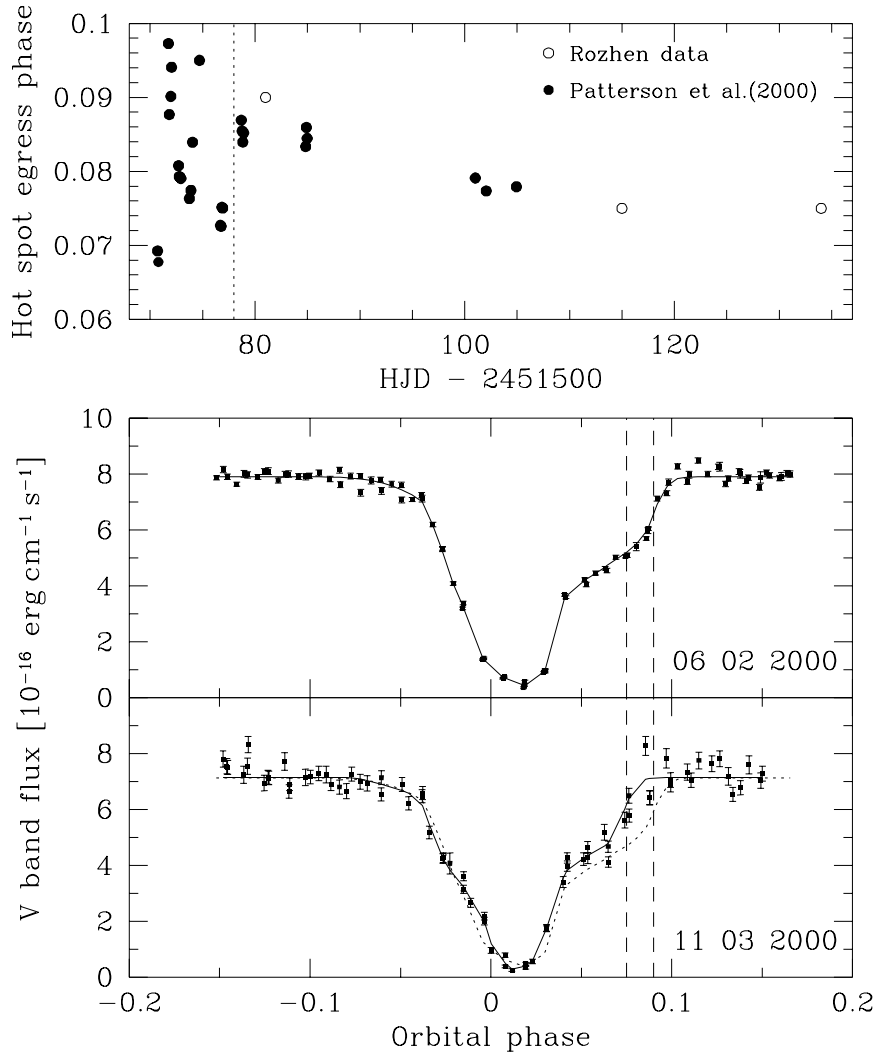

Fig. 2. Upper panel: hot spot egress phases. The trend after JD 2451578 indicates a decrease of accretion disc radius. Vertical line marks the end of late superhumps era. Lower panel: light curves of the reconstructed disc images. Dotted line is the reconstructed February light curve plotted over March data to see the difference clearly. Vertical dashed lines show the hot spot mid-egress phase

compact sources seem to be resolved, as was observed for example in Z Cha and OY Car (Wood et al. 1986, 1989). In Fig. 2 are also shown the mid-egress phases of the hot spot obtained from our data, compared with those derived by Patterson et al. (2000). It can be seen that our results are in agreement with a continuous decrease of the hot spot mid-egress phase when the system evolved to quiescence.

The calculated brightness distributions are shown in Fig. 3. Two compact sources are found and they are recognised as the white dwarf and the hot spot. The stream trajectories, calculated by integrating the equations of motion (Flannery 1975) by the fourth-order Runge-Kutta method, are also shown in Fig. 3. In both maps the hot spot is found close along the trajectory but at different distance from the white dwarf, indicating that the accretion disc has shrank. The disc radii in February and March, determined by the most bright pixel in the hot spot, are $r_{\mathrm{d}} \simeq 0.36 a$ and $r_{\mathrm{d}} \simeq 0.25 a$, respectively. In the disc reconstruction from the March data, something like a third spot is seen. We believe that this is not a real structure in the disc but is an artifact from the reconstruction.

Having the brightness distribution calculated we can estimate the brightness temperature $T_{\mathrm{BR}}$ of the accretion 

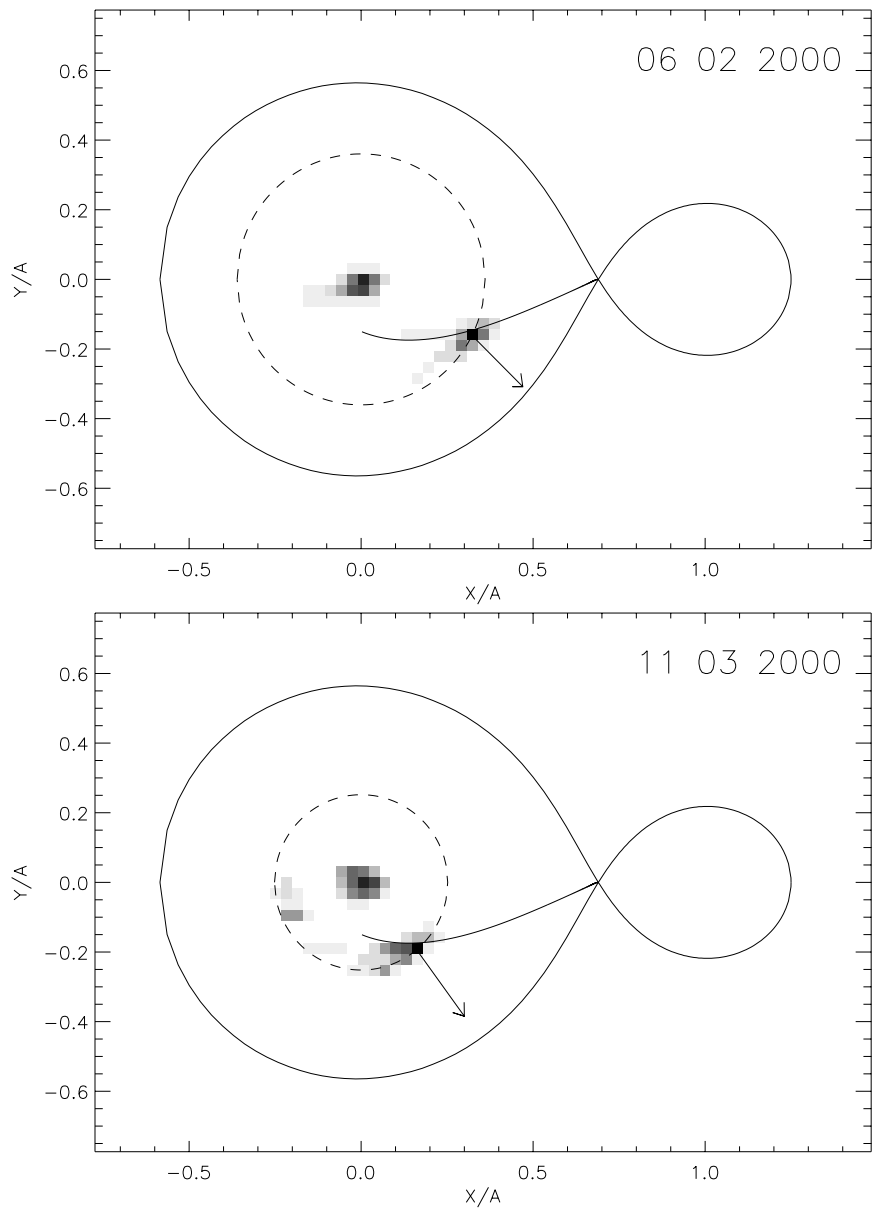

Fig. 3. Reconstructed brightness distribution of the accretion disc from February and March data. Arrows mark the direction of the maximal hot spot visibility

disc assuming black body emission. This is done by solving for $T_{\mathrm{BR}}$ the following equation:

$f=\frac{\int v(\lambda) B_{\lambda}\left(T_{\mathrm{BR}}\right) / \lambda \mathrm{d} \lambda}{\int v(\lambda) / \lambda \mathrm{d} \lambda} \delta / D^{2}$.

Here $f$ stands for the emitted flux, $B_{\lambda}\left(T_{\mathrm{BR}}\right)$ is the black body spectral distribution, $v(\lambda)$ is the response of the $V$ bandpass and $\delta$ is the pixel area corrected for the foreshortening. In Fig. 4 we show the radial distributions of the brightness temperature along the accretion disc radius together with the expected temperatures for steady accretion discs for different mass transfer rates. The inner part of the disc seems to be well described as a steady accretion disc model with $\dot{M} \simeq 10^{-11} M_{\odot} \mathrm{yr}^{-1}$. If this is the case it would be in conflict with other results for dwarf novae in quiescence where the accretion disc brightness temperature radial distribution is found to be much flatter than what is expected for steady discs. Most probably, however, this is redistributed flux from the white dwarf as a result of the algorithm. In the outer part of the disc reconstructed from the February data, the brightness temperature distribution becomes almost flat with $T_{\mathrm{BR}} \simeq 5500 \mathrm{~K}$. In the March data reconstruction this flat part cannot be seen due to the smaller disc radius and the redistribution of the

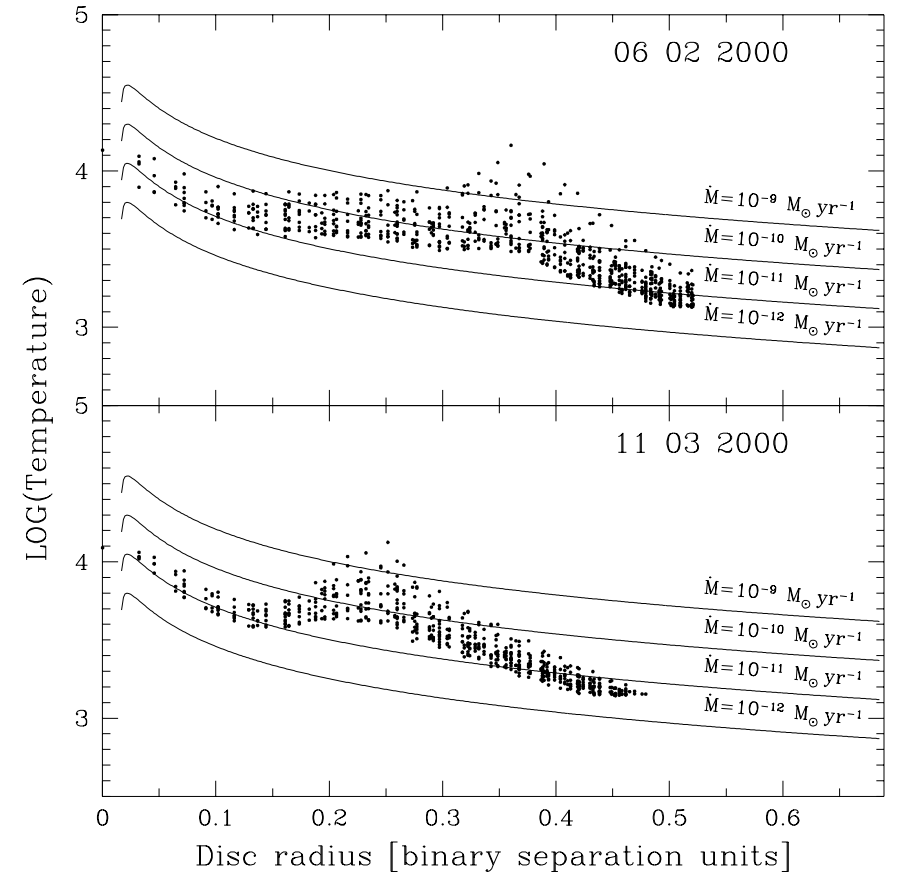

Fig. 4. Brightness temperature of the disc. Models of steady state discs for different mass transfer rates are shown also

white dwarf and the hot spot fluxes. While $T_{\mathrm{BR}} \simeq 5500 \mathrm{~K}$ is a typical temperature for dwarf novae in quiescence, the observations and numerical studies show that quiescent accretion discs in these systems are most probably optically thin. In this case the emission of the disc significantly deviates from black body law and the above value of $T_{\mathrm{BR}}$ is a very crude estimation of the effective temperature of the accretion disc.

The prominent humps seen roughly between phases -0.4 and +0.1 are believed to be produced by the hot spot. The hot spot light is assumed to be emitted by a circular area perpendicular to the orbital plane, and the hump is produced by the foreshortening (Wood et al. 1986, 1989). These authors supposed that the size of the spot is equal to the cross section of the accretion stream (the formula derived by Cook \& Warner (1984)) and used Eq. (1) to estimate $T_{\mathrm{BR}}$ of the spot assuming two models of hot spot emission:

i) the hot spot radiates isotropically from both its sides with most of the light from nearest to the disc side being obscured from our sight;

ii) a two component model in which the hot spot itself radiates anisotropically from its outer side only and another isotropic component.

Since we cannot decompose our light curves, we used this method to estimate $T_{\mathrm{BR}}$ of the spot corresponding to the anisotropic part in the second model. The flux $f$ was determined as the difference between maximal flux and the flux at phases when the light of the hot spot is not seen (in March data the fluxes before and after the hump are significantly different so we took the mean value). The estimations showed that the strength of the hump decreases 
roughly by $\sim 30 \%$ between February and March, giving $T_{\mathrm{BR}} \simeq 15900 \mathrm{~K}$ and $T_{\mathrm{BR}} \simeq 13800 \mathrm{~K}$ with an error of both values $\sim 500 \mathrm{~K}$. These values are an upper limit for $T_{\mathrm{BR}}$ because the accepted width of the hot spot is a lower limit. The actual size of the spot is probably much larger as it is produced by the hypersonic impact between the stream and disc.

\section{Conclusions}

The analysis of the data shows that in spite of the constant system brightness after February (Fig. 1 in Patterson et al. 2000, and our data) the accretion disc has not reached its equilibrium quiescent state. Its radius gradually decreases as the system evolves to quiescence from $r_{\mathrm{d}} \simeq 0.36 a$ in February to $r_{\mathrm{d}} \simeq 0.25 a$ in March. This is in agreement with both the observations and the numerical simulations of dwarf nova outbursts (see review paper of Osaki 1996, and references therein). In addition, the temperature of the disc itself seems to remain constant. The light curves obtained on 11 and 30 March are very similar without any apparent changes, which suggests that the equilibrium state was most probably reached in the beginning of March. The eclipse mapping shows a hot spot, laying along the accretion stream. The strength of the orbital hump decreases by $\sim 30 \%$. Assuming that the hump is produced by the hot spot and that it emits anisotropically from its outer side, we estimate an upper limit of the hot spot brightness temperature $T_{\mathrm{BR}} \simeq 15900 \mathrm{~K}$ and
$T_{\mathrm{BR}} \simeq 13800 \mathrm{~K}$ in February and March, respectively. Such a decrease could be produced by an enhanced mass transfer rate during outburst or/and by a change of the hot spot structure as the angle of impact and densities of the disc and stream vary with the accretion disc radius.

Acknowledgements. V.S, Z.K and V.G are grateful to NFSR for supporting this work with the project No. 715/97.

\section{References}

Cook, M. C., \& Warner, B. 1984, MNRAS, 207, 705

Flannery, B. P. 1975, MNRAS, 170, 325

Henden, A. A. 2000, vsnet-alert, 4053

Horne, K. 1985, MNRAS, 213, 129

Osaki, Y. 1996, PASP, 108, 39

Patterson, J., Kemp, J., Jensen, L., et al. 2000, PASP, 112, 1567

Samus, N. N. 2000, IAU Circ., 7353

Schmeer, N. N. 2000, vsnet-obs Circ., 4027

Skilling, J., \& Btyan, R. K. 1984, MNRAS, 211, 111

Stetson, P. 1987, PASP, 99, 191

Straižys, V. 1977, Multicolour Stellar Photometry: Photometric systems and methods (Mokslas Publishers, Vilnius, in Russian)

Takamizawa, K. 1998, vsnet-obs Circ., 18078

Wood, J., Horne, K., Berriman, G., et al. 1986, MNRAS, 219, 629

Wood, J., Horne, K., Berriman, G., \& Wade, R. 1989, ApJ, 341,974

Uemura, M., Kato, T., Matsumoto, K., et al. 2000, PASJ, 52, L9 